Second Meeting, 13th December 1901.

Dr ThIRd, Vice-President, in the Chair.

\title{
Note on the theory of the rolling of one rigid surface on another.
}

By R. F. Muirhead, M.A., B.Sc.

Take first the case of a surface $\mathrm{S}$ rolling on a plane, the instantaneous axis of rotation being a line in the tangent plane at the point of contact. Take that line as $x$-axis, and the normal as $z$-axis, and let the equation to the surface be

$$
z=a x^{2}+2 h x y+b y^{2}+\text { terms of higher degree in } x, y
$$

Then if $p$ is small, the equation of the section of the surface $\mathbf{S}$ by the plane $z=p$ is

$$
a x^{2}+2 h x y+b y^{2}=p
$$

Now for rolling of this sort it is clear that the successive axes of rotation during a short time will be generators parallel to $\mathrm{OX}$ of a cylindrical surface which will touch $\mathrm{S}$ in the neighbourhood of $O$. The surface will for a short time roll as if it were rigidly connected with the cylinder, and that particular generator of the cylinder which touches the curve (2) will presently be the instantaneous axis. Thus the point of contact of the tangent to (2) which is parallel to $\mathrm{OX}$ will then be the point in contact with the plane on which $S$ rolls. Hence the diameter of (2) which is conjugate to OX will give the direction of the tangent to the trace of the rolling surface on the plane.

Next consider the rolling of the surface $\mathbf{S}$ on another surface $\mathbf{S}^{\prime}$ whose equation is

$$
z=a^{\prime} x^{2}+2 h^{\prime} x y+b^{\prime} y^{2}+\text { terms of higher degree in } x, y \text { - }
$$

the axis of rolling being as before supposed to lie in the tangent plane at the point of contact, and the coordinate axes taken as before. 
Let us consider the sections of the surfaces $S$ and $S^{\prime}$ made by the plane $y=k$, where $k$ is small. It is clear that these sections will presently come into contact with one another, and that the point of contact will be that point of $\mathrm{S}$ whose $z$-coordinate differs least from that of the corresponding point of $S$ ', where by "corresponding points in $S$ and $S^{\prime}$ " are meant those that have the same values of $x$ and $y$.

Now let us set out from $O$ in a direction making an angle $\theta$ with $O X$, along the two surfaces until we reach points $\mathrm{P}, \mathrm{P}^{\prime}$ in the plane $y=k$ and $\mathrm{Q}, \mathrm{Q}^{\prime}$ in the plane $z=p$; so that $\mathrm{P}$ and $\mathrm{P}^{\prime}$ are "corresponding points," and $Q$ and $Q^{\prime}$ lie in (2) and (3) respectively.

The value of $z$ for $\mathrm{P}$ is clearly $p \cdot \mathrm{OP}^{2} / \mathrm{OQ}^{2}$, and for $\mathrm{P}^{\prime}$ it is $p \times \mathrm{OP}^{\prime 2} / \mathrm{OQ}^{\prime 2}$, where $\mathrm{OP}=\mathrm{OP}^{\prime}=k \operatorname{cosec} \theta$.

Thus the difference between the $z$ 's is

But by (2) and (3)

$$
p k^{2} \operatorname{cosec}^{2} \theta\left(1 / \mathrm{OQ}^{2}-1 / \mathrm{OQ}^{\prime \prime}\right)
$$

$$
\begin{aligned}
& \mathrm{OQ}^{2}\left(a \cos ^{2} \theta+2 h \cos \theta \sin \theta+b \sin ^{2} \theta\right)=p \\
& \mathrm{OQ}^{\prime 2}\left(a^{\prime} \cos ^{2} \theta+2 h^{\prime} \cos \theta \sin \theta+b^{\prime} \sin ^{2} \theta\right)=p .
\end{aligned}
$$

Hence (4) becomes

$$
\begin{aligned}
& k^{2} \operatorname{cosec}^{2} \theta\left\{\left(a-a^{\prime}\right) \cos ^{2} \theta+2\left(h-h^{\prime}\right) \cos \theta \sin \theta+\left(b-b^{\prime}\right) \sin ^{2} \theta\right\} \\
& \quad \text { or } k^{2}\left\{\left(a-a^{\prime}\right) \cot ^{2} \theta+2\left(h-h^{\prime}\right) \cot \theta+\left(b-b^{\prime}\right)\right\}
\end{aligned}
$$

Now if we consider the conic

$$
\left(a-a^{\prime}\right) x^{2}+2\left(h-h^{\prime}\right) x y+\left(b-b^{\prime}\right) y^{2}=\text { constant }
$$

which may be written

$$
y^{2}\left\{\left(a-a^{\prime}\right) \cot ^{2} \theta+2\left(h-h^{\prime}\right) \cot \theta+\left(b-b^{\prime}\right)\right\}=\text { constant }
$$

it is clear that (5) is least when $y$ is greatest in (7), i.e., for the direction conjugate to $O X$.

Thus the direction of the trace of rolling on $\mathbf{S}$ or $\mathbf{S}^{\prime}$ is that of the diameter conjugate to $\mathrm{OX}$ in the conic (6).

This we might interpret by the statement that the direction of the trace is the same as if a surface, whose $z$-coordinates in the neighbourhood of the point of contact were equal to the differences of the corresponding ordinates of the surfaces $S$ and $S^{\prime}$, were to roll on the tangent plane at $O$. This result is perhaps intuitively evident.

If we wished to state the results in terms of the Indicatrix, we 
might say that for a surface rolling without spin on a plane, the trace on the surface or on the plane has for its tangent the diameter of the indicatrix which is conjugate to the instantaneous axis of rolling; and for one surface rolling without spin on another the direction of the trace is that which is conjugate to the axis of rolling, in the conic whose asymptotes are the common diameters of the two indicatrices at the point of contact. (Of course, for surfaces not intersecting one another in the neighbourhood of the point of contact, these asymptotes would be imaginary.)

In the preceding we have taken as the standard case, that in which both surfaces lie on the same side of the tangent plane at their point of contact. The necessary modifications when they are on opposite sides are obvious.

We can now extend our results to the more general case of rolling combined with spinning. In that case the instantaneous axis is through the point of contact, but not in the tangent plane; and this rotation can be resolved into a spin about the common normal, and rolling about an axis which is the projection of the instantaneous axis upon the tangent plane. But it is clear that the presence of the spinning will, during a short time, canse only an infinitesimal rotation of the indicatrix of $S$, so that the preceding results will hold when we substitute for the instantaneous axis its projection on the tangent plane.

If we take the still more general case where sliding is present as well as rolling and spinning, it is not difficult to see that the direction of the trace left by $S$ on $S^{\prime}$ supposed at rest, would be got by compounding the velocity of the description of the trace on $S^{\prime}$ on the supposition of no sliding, with the velocity of sliding of $\mathbf{S}$ on $\mathbf{S}^{\prime}$ at the instant.

Lastly, if both surfaces, while keeping in contact, were in motion, the direction of the trace on either could be got by considering the motion of the other relative to it.

In Thomson and Tait's Natural Philosophy, Vol. I., Part I., $\$ \$ 110,111$, the subject of rolling is treated very fully, and equations are established from which the above results could easily be deduced, but $I$ have ventured to bring this note before the Society because the somewhat different treatment that I bave adopted seems simpler so far as it goes. 


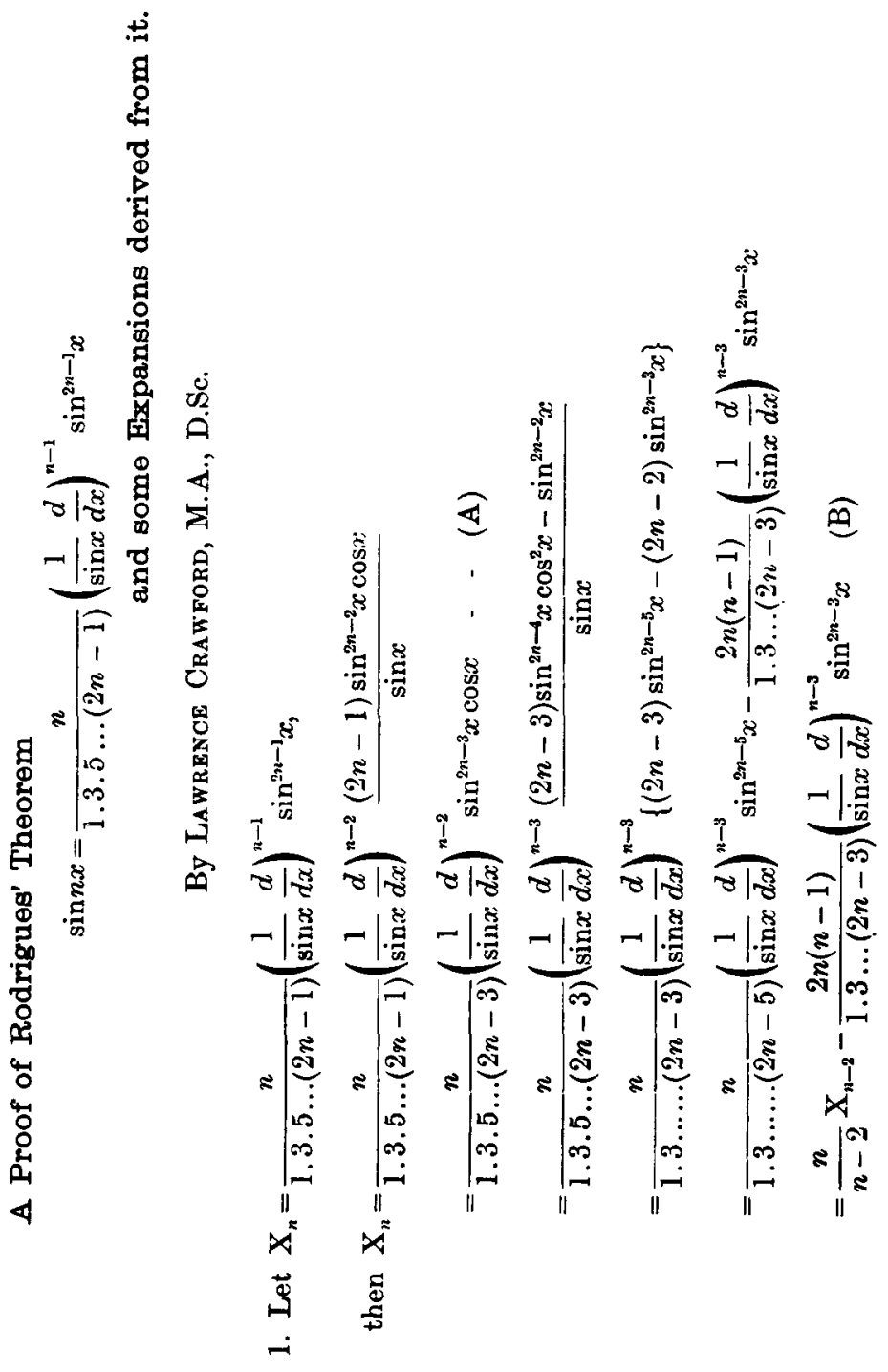




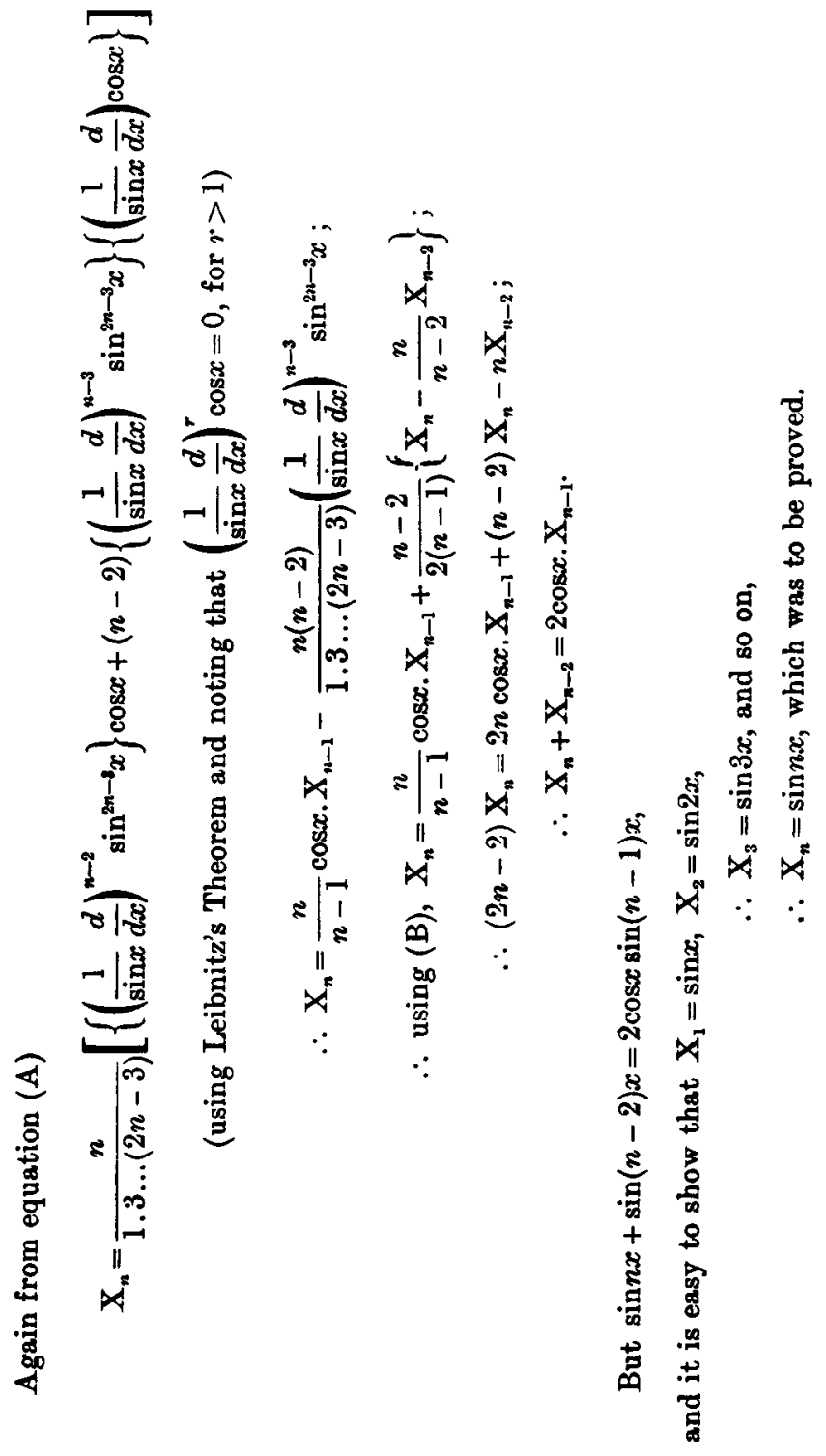




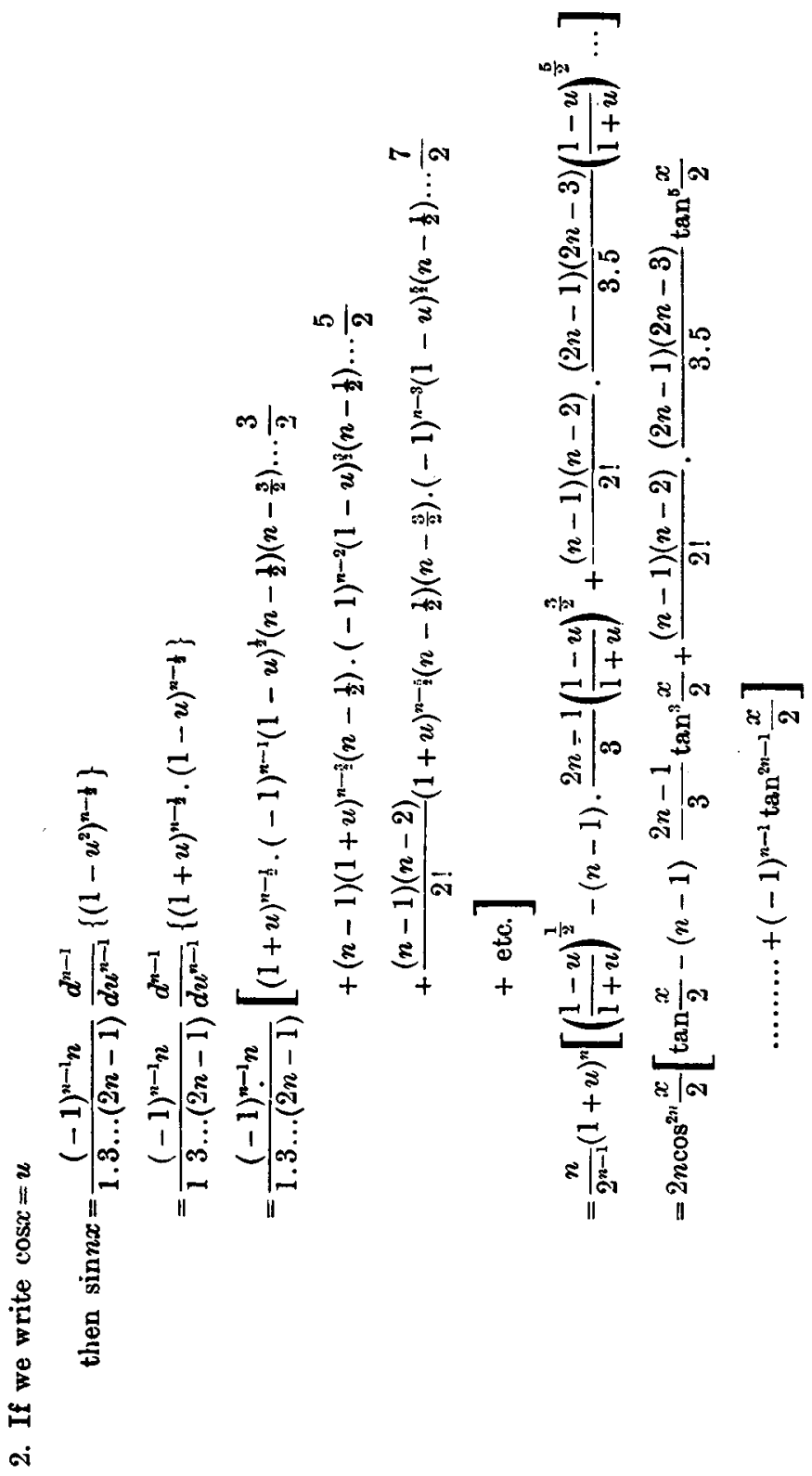

2 Vol. 20 

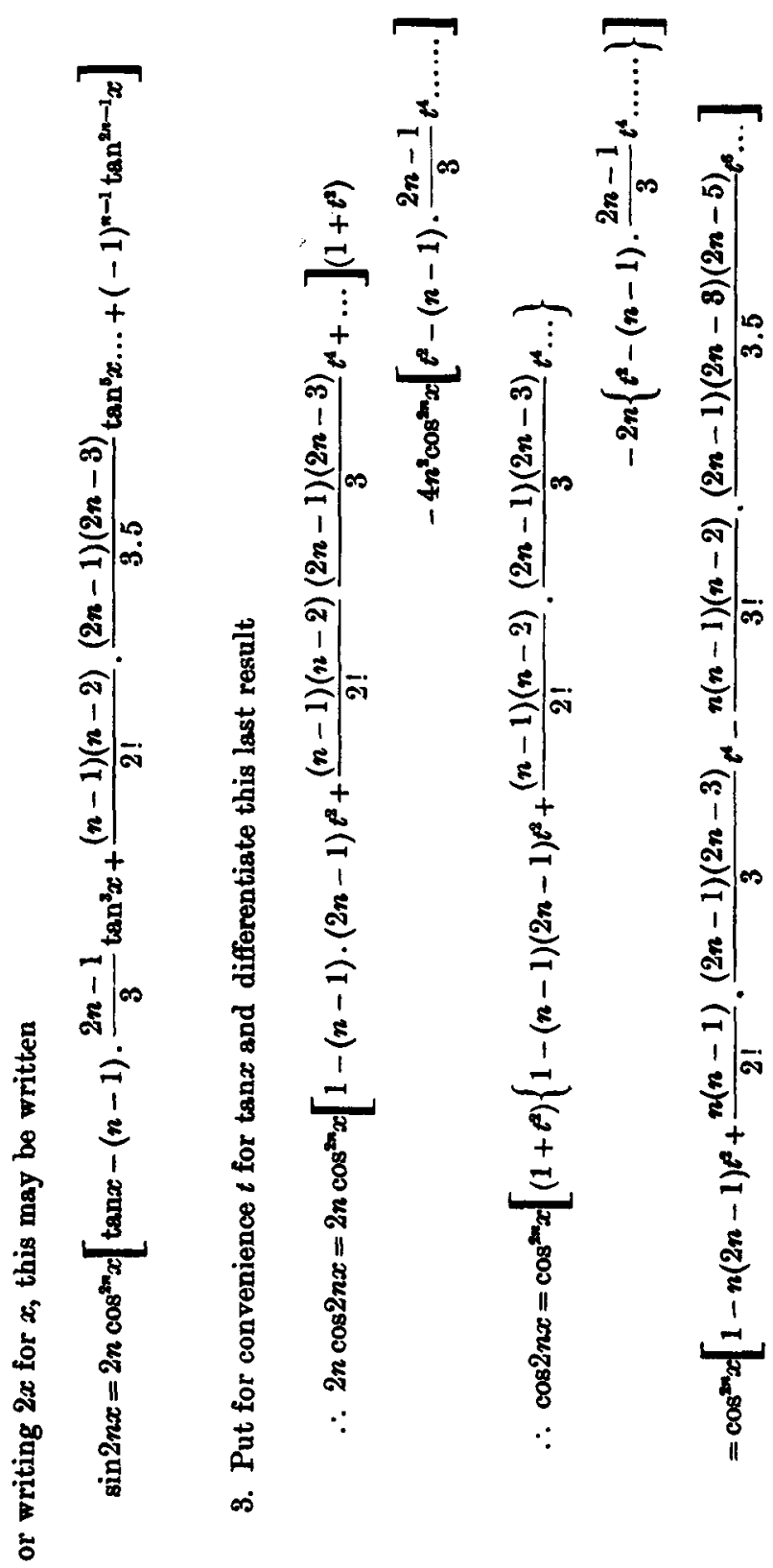


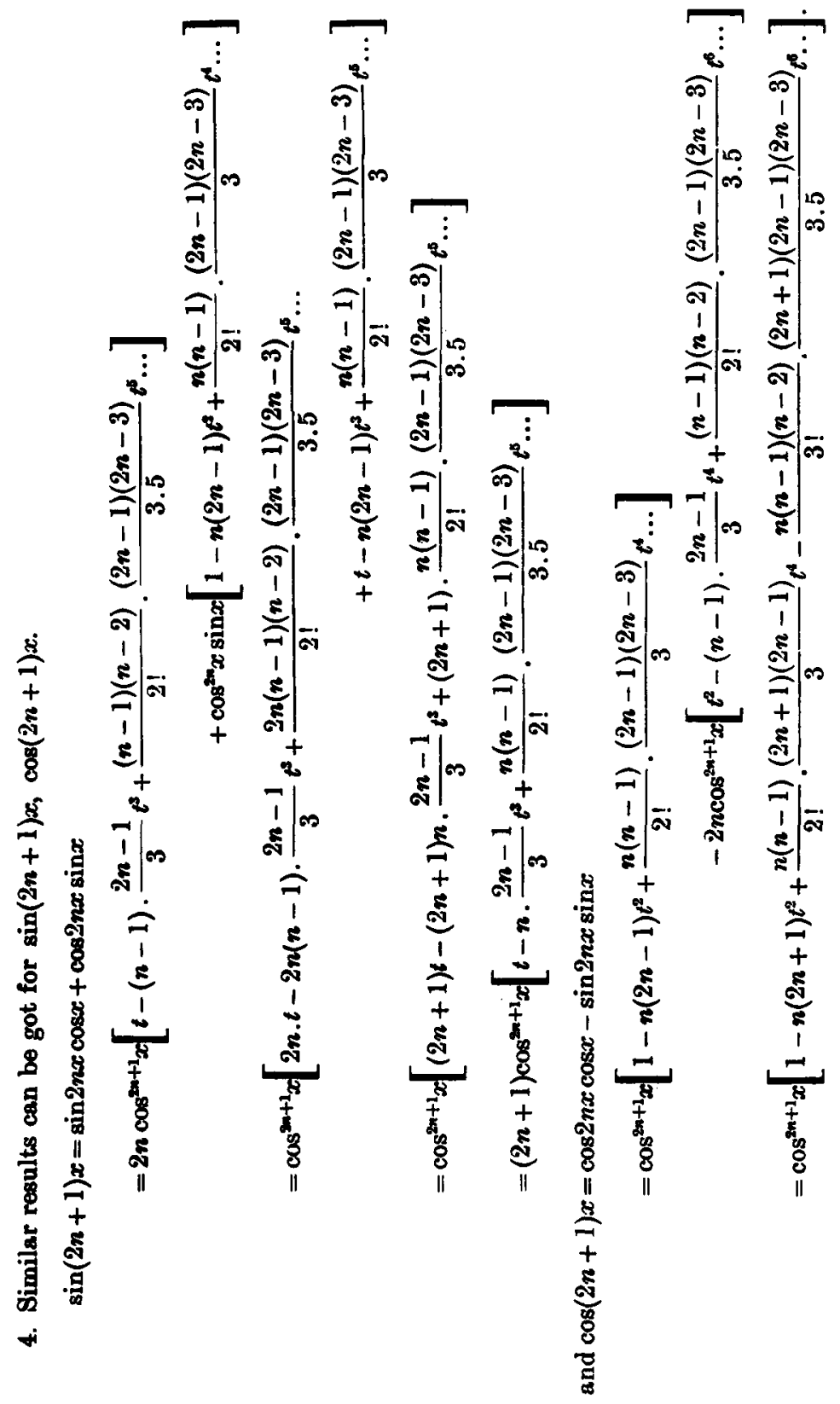

